Check for updates

Cite this: Phys. Chem. Chem. Phys., 2021, 23, 10321

Received 1st March 2021, Accepted 12th April 2021 DOI: $10.1039 / \mathrm{d} 1 \mathrm{cp} 00925 \mathrm{~g}$ rsc.li/pccp

\section{The effect of defect interactions on the reduction of doped ceria $\dagger$}

\author{
Steffen Grieshammer (iD) abc
}

Doped cerium oxide is known for its reduction properties that are utilized in catalytic applications as well as in thermochemical cycling to produce solar fuels. Upon reduction of the lattice, oxygen vacancies and polarons are formed leading to a highly concentrated solution of defects in which the interactions of defects cannot be neglected anymore. In this study, the effect of defect interactions on the free energy of reduction for doped ceria with composition $\mathrm{Ce}_{1-x-y} \mathrm{RE}_{x} \mathrm{Zr}_{y} \mathrm{O}_{2-x / 2-\delta}$ was investigated by large scale Metropolis Monte Carlo multi-stage sampling simulations based on first-principles calculations. The simulations allowed the prediction of the relation between oxygen partial pressure and non-stoichiometry for the highly interacting, non-ideal system. The results show that the non-ideality observed in experiments can be traced back to the interactions of defects and allow prediction of the reduction behavior for various dopant types, dopant fractions, temperatures, and non-stoichiometries.

\section{Introduction}

Cerium oxide (ceria) is an essential material in various applications such as solid oxide fuel cells, industrial and automotive catalysis, and more recently the production of solar fuels. ${ }^{1-5}$ One of the most prominent properties of ceria is its high oxygen storage capacity (OSC) that is utilized in catalytic reactions and the two step process to produce hydrogen from water. The OSC is based on the reduction equilibrium given in eqn (1) in Kröger-Vink-notation, where the release of oxygen gas leads to the creation of oxygen vacancies and polarons, i.e. localized electrons.

$$
2 \mathrm{Ce}_{\mathrm{Ce}}^{\times}+\mathrm{O}_{\mathrm{O}}^{\times} \rightleftharpoons 2 \mathrm{Ce}_{\mathrm{Ce}}^{\prime}+\frac{1}{2} \mathrm{O}_{2(\mathrm{~g})}+\mathrm{V}_{\mathrm{O}}^{\bullet \bullet}
$$

An even higher OSC is achieved by doping the material with rare-earth oxides $\left(\mathrm{RE}_{2} \mathrm{O}_{3}\right)$ or zirconium oxide $\left(\mathrm{ZrO}_{2}\right) \cdot{ }^{6-10}$ While aliovalent rare-earth doping (eqn (2)) leads to the formation of oxygen vacancies, isovalent $\mathrm{Zr}^{4+}$ introduces no additional defects (eqn (3)) but modifies the material's properties due to the smaller size of the $\mathrm{Zr}$-ions and the corresponding lattice distortions.

$$
\mathrm{RE}_{2} \mathrm{O}_{3} \stackrel{\mathrm{CeO}_{2}}{\longrightarrow} 2 \mathrm{RE}_{\mathrm{Ce}}^{\prime}+\mathrm{V}_{\mathrm{O}}^{\bullet \bullet}+3 \mathrm{O}_{\mathrm{O}}^{\times}
$$

\footnotetext{
${ }^{a}$ Institute of Physical Chemistry, RWTH Aachen University, Landoltweg 2, 52056 Aachen, Germany. E-mail: grieshammer@pc.rwth-aachen.de

${ }^{b}$ Helmholtz-Institut Münster (IEK-12), Forschungszentrum Jülich GmbH, Corrensstr. 46, 48149 Münster, Germany

c JARA-HPC, Germany, Forschungszentrum Jülich \& RWTH Aachen University, Germany

$\dagger$ Electronic supplementary information (ESI) available. See DOI: 10.1039/ d1cp00925g
}

$$
\mathrm{ZrO}_{2} \stackrel{\mathrm{CeO}_{2}}{\longrightarrow} \mathrm{Zr}_{\mathrm{Ce}}^{\times}+2 \mathrm{O}_{\mathrm{O}}^{\times}
$$

As the OSC and reduction behavior directly influence the efficiency of the catalytic reaction and production of solar fuels, understanding the impact of doping and defect interactions on these properties is crucial.

For an ideal, non-interacting system, the classical mass action law for eqn (1) can be expressed in terms of the molar fractions by,

$$
K_{0}(T)=\frac{\left[\mathrm{V}_{\mathrm{O}}^{\bullet \bullet}\right]\left[\mathrm{Ce}_{\mathrm{Ce}}^{\prime}\right]^{2}}{\left[\mathrm{O}_{\mathrm{O}}^{\times}\right]\left[\mathrm{Ce}_{\mathrm{Ce}}^{\times}\right]^{2}} p_{\mathrm{O}_{2}}^{1 / 2}=\exp \left(-\frac{\Delta_{\mathrm{r}} h^{\circ}}{k_{\mathrm{B}} T}+\frac{\Delta_{r} s^{\circ}}{k_{\mathrm{B}}}\right)
$$

where $\Delta_{\mathrm{r}} h^{\circ}$ and $\Delta_{\mathrm{r}} s^{\circ}$ are the standard enthalpy and entropy of reduction, respectively. This approach implies an ideal behavior of the defects without any defect interactions which is only true for the diluted case. In contrast, experimental investigations show a clearly non-ideal reduction behavior ${ }^{10-15}$ and computational studies have proven the influence of defect interactions on the energy of reduction. ${ }^{16-18}$

Therefore, the term $\Delta_{\mathrm{r}} g^{\text {int }}$ has to be considered, which is the contribution of defect interactions to the Gibbs energy of reduction per formula unit $\mathrm{CeO}_{2}$. Neglecting volume changes in the solid, the approximation $\Delta_{\mathrm{r}} g^{\mathrm{int}} \approx \Delta_{\mathrm{r}} f^{\text {nt }}$ is applicable. The deviation from the ideal reduction behavior can then be expressed by an excess term with the derivative of $\Delta_{\mathrm{r}} f^{\text {int }}$ for $\delta$ (see ESI $\dagger$ for details):

$$
K_{\text {int }}(T, \delta)=\exp \left(-\frac{\partial \Delta_{\mathrm{r}} f^{\text {int }}(T, \delta)}{\partial \delta} \frac{1}{k T}\right)
$$


For the general composition $\mathrm{Ce}_{1-x-y} \mathrm{RE}_{x} \mathrm{Zr}_{y} \mathrm{O}_{2-x / 2-\delta}$, we can write the charge and site balances as follows:

$$
\begin{aligned}
& {\left[\mathrm{V}_{\mathrm{O}}^{\bullet \bullet}\right]=\left(\left[\mathrm{Ce}_{\mathrm{Ce}}^{\prime}\right]+\left[\mathrm{RE}_{\mathrm{Ce}}^{\prime}\right]\right) / 2=\delta+x / 2} \\
& {\left[\mathrm{O}_{\mathrm{O}}^{\times}\right]=2-\left[\mathrm{V}_{\mathrm{O}}^{\bullet \bullet}\right]=2-x / 2-\delta} \\
& {\left[\mathrm{Ce}_{\mathrm{Ce}}^{\times}\right]=1-\left[\mathrm{RE}_{\mathrm{Ce}}^{\prime}\right]-\left[\mathrm{Zr}_{\mathrm{Ce}}^{\times}\right]-\left[\mathrm{Ce}_{\mathrm{Ce}}^{\prime}\right]=1-x-y-2 \delta}
\end{aligned}
$$

Inserting in eqn (4) and combining with eqn (5) results in

$$
K_{0}(T) K_{\text {int }}(T, \delta)=\frac{4 \delta^{2}(\delta+x / 2)}{(2-x / 2-\delta)(1-x-y-2 \delta)^{2}} p_{\mathrm{O}_{2}}{ }^{1 / 2}
$$

Rearranging yields the relationship between oxygen partial pressure $p_{\mathrm{O}_{2}}$ and non-stoichiometry $\delta$.

$$
p_{\mathrm{O}_{2}}=\left(\frac{(2-x / 2-\delta)(1-x-y-2 \delta)^{2}}{4 \delta^{2}(\delta+x / 2)} K_{0}(T) K_{\text {int }}(T, \delta)\right)^{2}
$$

It is noted that $K_{0}(T)$ solely depends on the temperature whereas $K_{\mathrm{int}}(T, \delta)$ depends on temperature and non-stoichiometry as well as dopant type and fraction.

Using the Metropolis Monte Carlo algorithm, the simulation of the defect distribution in equilibrium and the estimation of the internal energy is possible. As the direct evaluation of the free energy contribution is not possible, Valleau and Card ${ }^{19}$ suggested a multistage sampling approach which connects the desired distribution $\left(T=T_{\min }\right)$ with the random distribution $(T=\infty)$ by a series of consecutive distributions $i$ with the temperature factor $\alpha_{i}=T_{\min } / T_{i}$. This approach allows the evaluation of $\Delta_{\mathrm{r}} \mathrm{f}^{\text {jnt }}$ with respect to the ideal solution and was successfully applied in the description of the non-ideal behavior of pure and Gd-doped ceria before. ${ }^{17}$ More details are given in the $\mathrm{ESI} \dagger$ and the according references.

In this paper, the effect of defect interactions on the free energy of reduction is investigated for various dopants and compositions in $\mathrm{Ce}_{1-x-y} \mathrm{RE}_{x} \mathrm{Zr}_{y} \mathrm{O}_{2-x / 2-\delta}$ using Metropolis Monte Carlo multi-stage sampling simulations based on density functional theory calculations. With the results, the deviation of the relation $\delta$ vs. $p_{\mathrm{O}_{2}}$ from the ideal behavior is investigated.

\section{Simulation setup}

Simulations were performed with the program MOCASSIN using the built in multi-stage sampling routine as described in the ref. 2 and 21. The interactions of defects, i.e. oxygen vacancies, polarons and dopant ions, were evaluated as a sum over the number of interactions $N_{i j}^{\mathrm{d}}$ and the corresponding pair interaction energies $\varepsilon_{i j}^{\mathrm{d}}$ for defect pairs $i j$ at distance $d$.

$$
E^{\mathrm{int}}=\sum_{d, i, j \leq i} N_{i j}^{d} \varepsilon_{i j}^{d}
$$

The pair interaction model is an approximation that neglects manybody interactions. Nevertheless, previous simulations have demonstrated the predictive power of this approach in ceria ${ }^{17,22-24}$ and the inclusion of many-body clusters would considerably increase the amount of DFT calculations taking into account the possible permutations of the polaron, rare-earth, and zirconium defects.

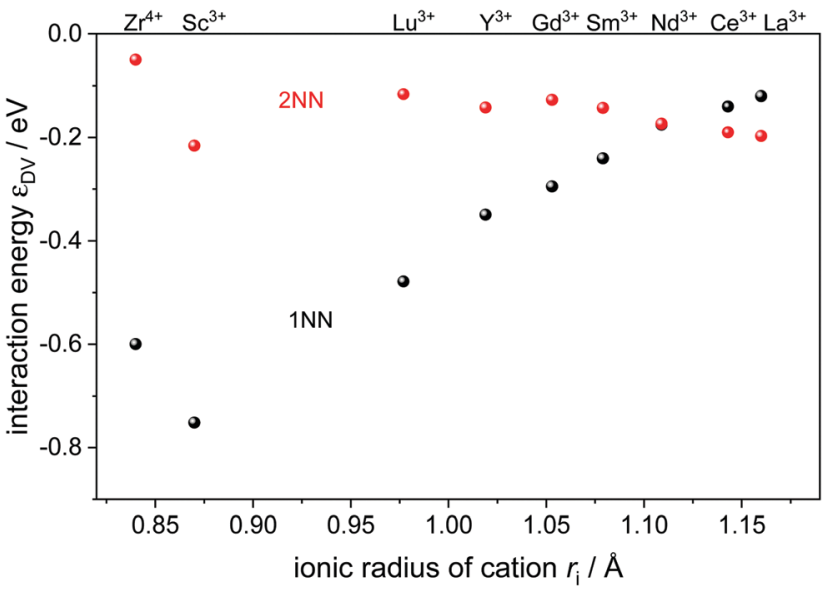

Fig. 1 Interaction energy $\varepsilon_{\mathrm{DV}_{\mathrm{O}}}^{\mathrm{d}}$ between an oxygen vacancy and a dopant ion or polaron in the nearest $(1 \mathrm{NN})$ and next nearest $(2 \mathrm{NN})$ neighbor position taken from ref. 16 and 18 .

The relevant pair interaction energies from density functional theory (DFT) calculations were taken from ref. 16 and 18 as listed in the ESI. $\dagger$ These energies were obtained with the PBE functional with an on-site Coulomb interaction on cerium f-orbitals of $U_{\text {eff }}=5 \mathrm{eV}$. It is noted that the choice of the functional influences the values of the interactions but significant changes are not expected. $^{24,25}$ Therefore, the general results of the simulations are expected to be widely independent of the applied functional.

In this study, typical rare-earth dopants for ceria were included which are distinguished by their ionic radii (Fig. 1). In addition, $\mathrm{Zr}^{4+}$ was included as a typical dopant and impurity in ceria.

The most relevant energies are the interactions between the oxygen vacancies and the dopant ions or polarons which are represented in Fig. 1. All interactions are negative implying attraction between the cation defects and oxygen vacancies. While the interaction in next nearest neighbor (2NN) position is similar for all cations, the interaction in nearest neighbor (1NN) position depends on the ionic radius. The strength of the $1 \mathrm{NN}$ interaction decreases with increasing cation radius which can be explained by the tendency of smaller cations to favor a lower coordination number. This results in a strong attraction for $\mathrm{Zr}_{\mathrm{Ce}}^{\times}-\mathrm{V}_{\mathrm{O}}^{\bullet}$ and $\mathrm{Sc}_{\mathrm{Ce}}^{\prime}-\mathrm{V}_{\mathrm{O}}^{\bullet \bullet}$ pairs that weakens for the other dopants. It is noted, that up to $\mathrm{Sm}^{3+}$ the $1 \mathrm{NN}$ position is energetically favorable compared to the $2 \mathrm{NN}$ position, whereas for $\mathrm{Nd}^{3+}$ both positions are energetically equal. For the polaron and for $\mathrm{La}^{3+}$ the $2 \mathrm{NN}$ position is most favorable.

Simulations were conducted in $12 \times 12 \times 12$ supercells with a total of 20736 lattice positions. The dopant ions were randomly distributed and considered as immobile due to their low diffusivity. ${ }^{26}$ In contrast, polarons and oxygen vacancies are highly mobile and $\mathrm{Ce}_{\mathrm{Ce}}^{\times} / \mathrm{Ce}_{\mathrm{Ce}}^{\prime}$ and $\mathrm{V}_{\mathrm{O}}^{\bullet \bullet} / \mathrm{O}_{\mathrm{O}}^{\times}$pairs were thus swapped during the simulations to obtain their equilibrium distribution. The temperature factor $\alpha$ was varied with a step size of 0.01 between 0 and 1 with $T_{\min }=773 \mathrm{~K}$.

The interaction contribution $\Delta_{\mathrm{r}} u^{\text {int }}$ to the internal energy of reduction was obtained from the simulation average of the energy states in thermodynamic equilibrium. The interaction contribution $\Delta_{\mathrm{r}} f^{\text {int }}$ to the free energy was obtained from the energy 
distributions collected in the multi-stage sampling approach (see ref. 17 or the ESI $\dagger$ for details). The entropy term $\Delta_{\mathrm{r}} s^{\text {int }}$ was calculated according to the relation $S=(U-F) / T$. All contributions were referenced to the stoichiometric case, thus reflecting only the contributions of defect interactions to the reduction of the lattice.

The non-stoichiometry was varied with a step size of 0.002 in the range $\delta \in[0.001 \ldots 0.009]$ and 0.01 in the range $\delta \in[0.01 \ldots 0.12]$. For each simulation, $10^{7}$ cycle steps were performed for equilibration of the lattice and energy states were recorded over $10^{8}$ cycle steps. Twelve independent simulations with different dopant distributions were averaged for each data point. These simulation parameters are in accordance with previous simulations. ${ }^{17}$

\section{Results and discussion}

\section{Energy contributions}

The simulated interaction contributions $\Delta_{\mathrm{r}} u^{\mathrm{int}}, \Delta_{\mathrm{r}} f^{\mathrm{nt}}$, and $\Delta_{\mathrm{r}} s^{\mathrm{int}}$ per formula unit $\mathrm{CeO}_{2}$ are given in Fig. 2. Internal energy $\Delta_{\mathrm{r}} u^{\text {int }}$ and free energy $\Delta_{\mathrm{r}} f^{\text {int }}$ contributions are negative due to the attractive interactions between the oxygen vacancies and cation defects. The energy values become more negative with increasing non-stoichiometry as the amount of defects and thus the number of defect interactions increases. This implies that $K_{\mathrm{int}}>1$ according to eqn (5), thus facilitating the reduction of the lattice.

The absolute value of the free energy is smaller than the absolute value of the internal energy, corresponding to the negative values of the entropy contribution. This is in accordance with the fact that any kind of interaction leads to an ordering of defects and thus a loss of configurational entropy compared to the ideal solution model.

Two different groups of dopants can be identified from the energies in Fig. 2 in correspondence with Fig. 1. The values are

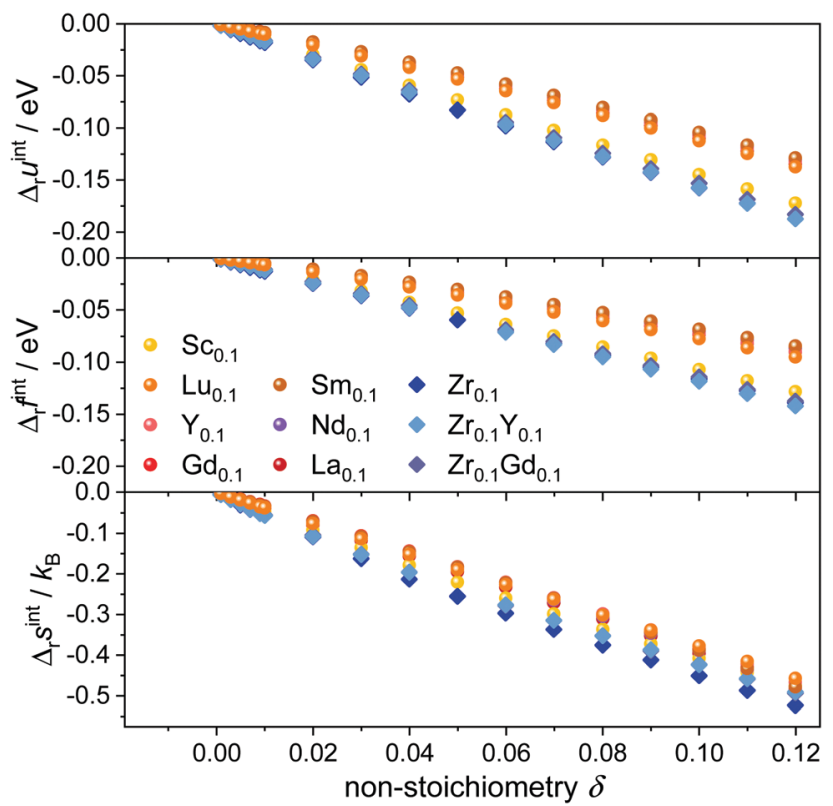

Fig. 2 Interaction contributions per formula unit to internal energy (top), free energy (center), and entropy (bottom) at $1073 \mathrm{~K}$ in $\mathrm{Ce}_{1-x-y} \mathrm{RE}_{x} \mathrm{Zr}_{y} \mathrm{O}_{2-x / 2-\delta}$ for various dopants. similar for the RE-dopants $\mathrm{Lu}^{3+}, \mathrm{Y}^{3+}, \mathrm{Gd}^{3+}, \mathrm{Sm}^{3+}, \mathrm{Nd}^{3+}$, and $\mathrm{La}^{3+}$ but considerably more negative for $\mathrm{Sc}^{3+}$ and $\mathrm{Zr}^{4+}$. Previous investigations revealed that the lowering of the energy during reduction is mainly due to the attractive interaction between the polarons and oxygen vacancies in $2 \mathrm{NN}$ position that is independent of the dopant type. ${ }^{16}$ A secondary effect is the interaction between the dopant ions and the oxygen vacancies introduced through reduction. This effect is less important for $\mathrm{Lu}^{3+}, \mathrm{Y}^{3+}, \mathrm{Gd}^{3+}, \mathrm{Sm}^{3+}, \mathrm{Nd}^{3+}$, and $\mathrm{La}^{3+}$ and consequently the results in Fig. 2 are similar. In contrast, the effect is more pronounced for $\mathrm{Sc}^{3+}$ due to a considerably stronger $\mathrm{Sc}_{\mathrm{Ce}}^{\prime}-\mathrm{V}_{\mathrm{O}}^{\bullet \bullet}$ interaction. For $\mathrm{Zr}^{4+}$, this effect is even more pronounced due the fact that no oxygen vacancies are present in the stoichiometric case. This leads to the formation of favorable $\mathrm{Zr}_{\mathrm{Ce}}^{\times}-\mathrm{V}_{\mathrm{O}}^{\bullet \bullet}$ pairs during reduction and a strong decrease of the energy. ${ }^{18}$ It should be noted at this point that the composition $\mathrm{Ce}_{0.9} \mathrm{Sc}_{0.1} \mathrm{O}_{1.95-\delta}$ is a hypothetical case as the Sc-concentration is above the solubility limit of a few percent. ${ }^{23}$

\section{Dependence of non-stoichiometry on oxygen partial pressure}

The relations between oxygen partial pressure and non-stoichiometry in doped ceria were obtained from eqn (8) with the respective values $K_{\text {int }}$ from eqn (5) and the experimentally determined values $\Delta_{\mathrm{r}} h^{\circ}=5 \mathrm{eV}$ and $\Delta_{\mathrm{r}} s^{\circ}=17 k_{\mathrm{B}}$ for the diluted case as described in the literature. ${ }^{11,17}$ The results for the various dopants at $1073 \mathrm{~K}$ are presented in Fig. 3 together with the values for pure ceria and the ideal curves for pure ceria and $\mathrm{Ce}_{0.9} \mathrm{RE}_{0.1} \mathrm{O}_{1.95-\delta}$ according to eqn (4). Compared to the ideal behavior, all curves are shifted by 4 to 15 orders of magnitude to higher partial pressures for a given non-stoichiometry. This implies a higher reducibility due to the existence of defect interactions. As the slopes of $\Delta_{\mathrm{r}} f^{\text {int }}$ in Fig. 2 are negative, $K_{\text {int }}>1$ according to eqn (5) leading to higher values of $p_{\mathrm{O}_{2}}$ for a given value of $\delta$ according to eqn (8).

Again, the two groups of dopants can be distinguished. The RE-dopants $\mathrm{Lu}^{3+}, \mathrm{Y}^{3+}, \mathrm{Gd}^{3+}, \mathrm{Sm}^{3+}, \mathrm{Nd}^{3+}$, and $\mathrm{La}^{3+}$ with medium

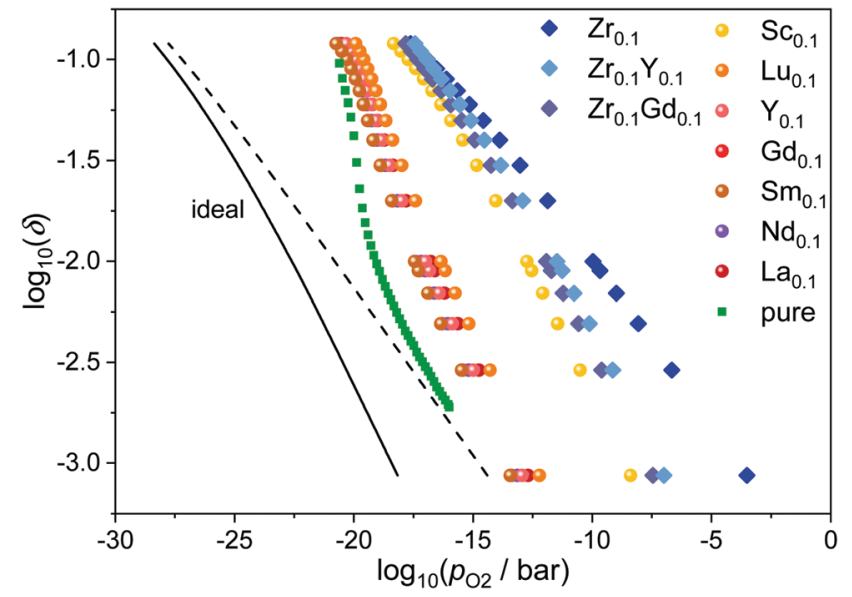

Fig. 3 Relation between oxygen partial pressure and non-stoichiometry in $\mathrm{Ce}_{1-x-y} \mathrm{RE}_{x} \mathrm{Zr}_{y} \mathrm{O}_{2-x / 2-\delta}$ at $1073 \mathrm{~K}$. The ideal behavior for $\mathrm{CeO}_{2-\delta}$ (dashed) and $\mathrm{Ce}_{0.9} \mathrm{RE}_{0.1} \mathrm{O}_{1.95-\delta}$ (solid) according to eqn (4) is indicated by the black line. Simulated values for pure ceria (squares) are taken from ref. 17. 
interactions show similar results while the $\mathrm{Sc}^{3+}$ and $\mathrm{Zr}^{4+}$ lead to reduction at considerably higher partial pressures. This reflects the finding, that doping with $\mathrm{Sc}^{3+}$ and $\mathrm{Zr}^{4+}$ leads to lower values of $\Delta_{\mathrm{r}} f^{\text {int }}$.

In addition, the slopes for $\mathrm{Sc}^{3+}$ and $\mathrm{Zr}^{4+}$ are flattened compared to the other dopants, meaning that the effect of interactions is more pronounced at low non-stoichiometries while the curves approach the other RE-dopants at high non-stoichiometries. This effect is likely to originate from a saturation effect: The strong attraction between vacancies and dopants leads to an ordering of vacancies close to the dopant ions even at low non-stoichiometry. At high non-stoichiometries, these favorable positions are already occupied and additional vacancies do not increase the number of favorable interactions considerably.

Fig. 3 also shows the effect of co-doping zirconia doped ceria with a rare-earth oxide. Comparing $\mathrm{Ce}_{0.9} \mathrm{Zr}_{0.1} \mathrm{O}_{2-\delta}$ with $\mathrm{Ce}_{0.8^{-}}$ $\mathrm{RE}_{0.1} \mathrm{Zr}_{0.1} \mathrm{O}_{1.95-\delta}$, it is apparent that the co-doping with $\mathrm{Y}_{2} \mathrm{O}_{3}$ or $\mathrm{Gd}_{2} \mathrm{O}_{3}$ leads to a shift of the curves to lower partial pressures, i.e. lower reducibility. This phenomenon can be attributed to two effects, which are due to the introduction of oxygen vacancies through RE-doping in the stoichiometric case. 1 . The minor effect is that the overall fraction of vacancies is increased and the fraction of oxygen ions is decreased leading to a smaller non-exponential factor in eqn (8). 2. The more important effect is that the oxygen vacancies order at the Zr-ions already in the stoichiometric, co-doped case, thus blocking these favorable positions and making the further creation of vacancies by reduction less favorable.

From the position of the curves in Fig. 3, an increase of the reducibility in the order pure ceria $<$ RE-doped $<$ Zr/RE-codoped $<$ Zr-doped can be inferred. This is well in line with experimental findings. ${ }^{7,27}$

The influence of the dopant fraction is illustrated in Fig. 4 for $\mathrm{Ce}_{1-x} \mathrm{Gd}_{x} \mathrm{O}_{2-x / 2-\delta}$ at $1073 \mathrm{~K}$. In general, the curves are shifted to higher oxygen partial pressures with increasing dopant fraction as expected for an increasing amount of favorable interactions. However, above 20 at $\%$ doping, the effect seems to diminish and furthermore, the curves approach each other at high non-stoichiometries. Both observations are likely due to the saturation effect mentioned above: As the number of defects

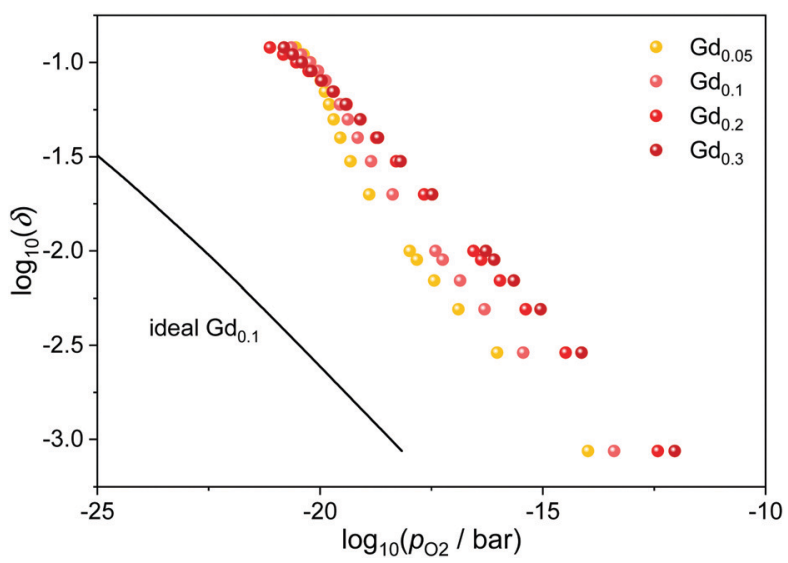

Fig. 4 Relation between oxygen partial pressure and non-stoichiometry in $\mathrm{Gd}$-doped ceria at $1073 \mathrm{~K}$ for different dopant fractions. The ideal behavior for $\mathrm{Ce}_{0.9} \mathrm{Gd}_{0.1} \mathrm{O}_{1.95-\delta}$ according to eqn (4) is indicated by the black line.

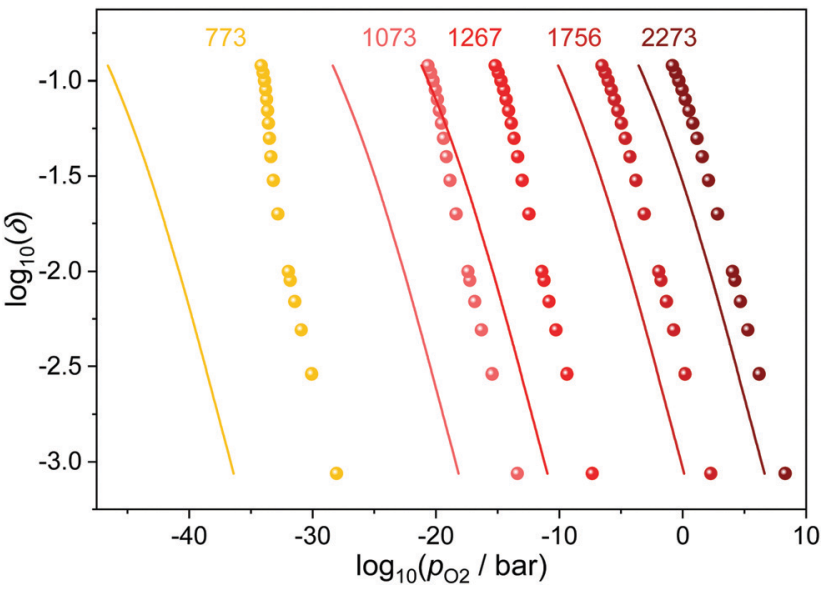

Fig. 5 Relation between oxygen partial pressure and non-stoichiometry in $\mathrm{Ce}_{0.9} \mathrm{Gd}_{0.1} \mathrm{O}_{1.9-\delta}$ for different temperatures. The ideal behavior according to eqn (4) is indicated by the respective lines.

increases, the energetically favorable configurations are already occupied, leading to a diminished effect for the further reduction.

The temperature influence for $\mathrm{Ce}_{0.9} \mathrm{Gd}_{0.1} \mathrm{O}_{1.95-\delta}$ is illustrated in Fig. 5. While all curves shift to higher partial pressures with increasing temperature, the differences between ideal and corrected curve diminish. This is due to the fact that high temperatures decrease the ordering of defects and thus the number of interacting pairs lowers. As a consequence, the impact of the defect interactions on the reduction of ceria is especially pronounced at low temperatures.

In the presented simulations, the dopant ions were distributed randomly, corresponding to the situation that can be expected for the slowly diffusing cations after quenching from high sintering temperatures. Nevertheless, long operation at elevated temperatures could lead to ordering of the dopant ions. Therefore, simulations were conducted with the dopant ions mobile, i.e. the ions are swapped during the simulations and allowed to reach their equilibrium distribution. The results are shown in Fig. 6 for

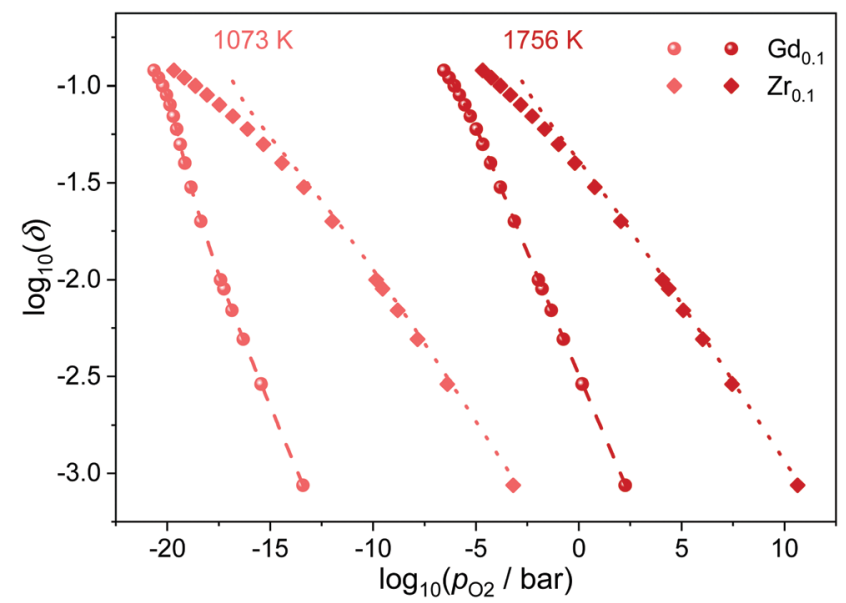

Fig. 6 Relation between oxygen partial pressure and non-stoichiometry in $\mathrm{Ce}_{0.9} \mathrm{Gd}_{0.1} \mathrm{O}_{1.9-\delta}$ and $\mathrm{Ce}_{0.9} \mathrm{Zr}_{0.1} \mathrm{O}_{2-\delta}$ at $1073 \mathrm{~K}$ and $1756 \mathrm{~K}$. Symbols represent the results for ordered $\mathrm{Gd}$ (spheres) and $\mathrm{Zr}$ (diamonds); lines represent the results for the random distribution of Gd (dashed) and $\mathrm{Zr}$ (dotted). 
$\mathrm{Ce}_{0.9} \mathrm{Gd}_{0.1} \mathrm{O}_{1.9-\delta}$ and $\mathrm{Ce}_{0.9} \mathrm{Zr}_{0.1} \mathrm{O}_{2-\delta}$ at $1073 \mathrm{~K}$ and $1756 \mathrm{~K}$. It is apparent that the ordering has no effect on the reduction behavior of Gd-doped ceria. The same is true for Zr-doping except for a little deviation at high non-stoichiometries. Overall, the ordering of defects seems to have only little effect on the reduction, independent of temperature and dopant type.

\section{Conclusions}

The impact of defect interactions on the reduction behavior of doped ceria with the general composition $\mathrm{Ce}_{1-x-y} \mathrm{RE}_{x} \mathrm{Zr}_{y} \mathrm{O}_{2-x / 2-\delta}$ was investigated by large scale Metropolis Monte Carlo multi-stage sampling simulations based on DFT energies. The contributions of defect interactions $\Delta_{\mathrm{r}} f^{\text {int }}$ to the free energy of reduction were estimated and the relation between oxygen partial pressure and non-stoichiometry for the non-ideal system was predicted. The results show a clear enhancement of the reduction due to defect interactions, leading to a shift of the partial pressure to higher values by orders of magnitude. In line with experimental results, an enhancement of the non-stoichiometry in the order pure ceria $<$ RE-doped $<\mathrm{Zr}$ /RE-co-doped $<\mathrm{Zr}$-doped is found and the following conclusions can be drawn:

- For a wide range of RE-dopants $\left(\mathrm{Lu}^{3+}\right.$ to $\left.\mathrm{La}^{3+}\right)$ the effect on the reduction is similar as the main influence is the interaction between polaron and oxygen vacancy.

- For $\mathrm{Sc}^{3+}$ the effect is more pronounced as the strong $\mathrm{Sc}_{\mathrm{Ce}}^{\prime}-\mathrm{V}_{\mathrm{O}}^{\bullet \bullet}$ interaction plays a more important role.

- The effect is even more pronounced for $\mathrm{Zr}^{4+}$ where the formation of oxygen vacancies during reduction leads to favorable $\mathrm{Zr}_{\mathrm{Ce}}^{\times}-\mathrm{V}_{\mathrm{O}}^{\bullet \bullet}$ pairs.

- The effect of interactions is especially pronounced at low temperatures, leading to stronger reduction compared to the ideal case.

- Additional co-doping of $\mathrm{Zr}$-doped ceria with $\mathrm{Gd}_{2} \mathrm{O}_{3}$ or $\mathrm{Y}_{2} \mathrm{O}_{3}$ decreases the reducibility due to the vacancies introduced in the stoichiometric composition.

- Above $\delta>0.1$, the reduction of the lattice seems to become progressively harder irrespective of the type of dopant or the dopant fraction.

- The ordering of dopant ions seems to have only little effect on the reduction behavior.

The simulation approach allows the prediction of the deviation from the ideal reduction behavior for a broad set of dopant types, dopant fractions, temperatures, and non-stoichiometries. Although the approach, based on pair interaction energies, is relatively simple, the simulations are in good agreement with the experimentally measured trends and values.

\section{Conflicts of interest}

There are no conflicts to declare.

\section{Acknowledgements}

The author gratefully acknowledges the computing time granted by the JARA Vergabegremium and provided on the
JARA Partition part of the supercomputer CLAIX at RWTH Aachen University.

\section{Notes and references}

1 H. Yao, J. Catal., 1984, 86, 254-265.

2 W. C. Chueh, C. Falter, M. Abbott, D. Scipio, P. Furler, S. M. Haile and A. Steinfeld, Science, 2010, 330, 1797-1801.

3 J. Kašpar, P. Fornasiero and M. Graziani, Catal. Today, 1999, 50, 285-298.

4 B. C. H. Steele, Solid State Ionics, 2000, 129, 95-110.

5 R. Schmitt, A. Nenning, O. Kraynis, R. Korobko, A. I. Frenkel, I. Lubomirsky, S. M. Haile and J. L. M. Rupp, Chem. Soc. Rev., 2020, 49, 554-592.

6 F. Call, M. Roeb, M. Schmücker, H. Bru, D. Curulla-Ferre, C. Sattler and R. Pitz-Paal, Am. J. Anal. Chem., 2013, 04, 37-45.

7 F. Call, M. Roeb, M. Schmücker, C. Sattler and R. Pitz-Paal, J. Phys. Chem. C, 2015, 119, 6929-6938.

8 A. Le Gal, S. Abanades and G. Flamant, Energy Fuels, 2011, 25, 4836-4845.

9 A. Le Gal, S. Abanades, N. Bion, T. Le Mercier and V. Harlé, Energy Fuels, 2013, 27, 6068-6078.

10 Y. Hao, C.-K. Yang and S. M. Haile, Chem. Mater., 2014, 26, 6073-6082.

11 R. J. Panlener, R. N. Blumenthal and J. E. Garnier, J. Phys. Chem. Solids, 1975, 36, 1213-1222.

12 S. Wang, Solid State Ionics, 1998, 107, 73-79.

13 S. R. Bishop, K. L. Duncan and E. D. Wachsman, Electrochim. Acta, 2009, 54, 1436-1443.

14 M. Kuhn, S. R. Bishop, J. L. M. Rupp and H. L. Tuller, Acta Mater., 2013, 61, 4277-4288.

15 G. Zhou, P. R. Shah, T. Kim, P. Fornasiero and R. J. Gorte, Catal. Today, 2007, 123, 86-93.

16 S. Grieshammer, M. Nakayama and M. Martin, Phys. Chem. Chem. Phys., 2016, 18, 3804-3811.

17 S. Grieshammer and M. Martin, J. Mater. Chem. A, 2017, 5, 9241-9249.

18 S. Grieshammer, J. Phys. Chem. C, 2017, 121, 15078-15084.

19 J. P. Valleau, J. Chem. Phys., 1972, 57, 5457.

20 S. Eisele and S. Grieshammer, J. Comput. Chem., 2020, 41, 2663-2677.

21 S. Eisele, F. M. Draber and S. Grieshammer, Phys. Chem. Chem. Phys., 2021, 23, 4882-4891.

22 S. Grieshammer, S. Eisele and J. Koettgen, J. Phys. Chem. C, 2018, 122, 18809-18817.

23 S. Grieshammer, B. O. H. Grope, J. Koettgen and M. Martin, Phys. Chem. Chem. Phys., 2014, 16, 9974.

24 P. A. Zguns, A. V. Ruban and N. V. Skorodumova, Phys. Chem. Chem. Phys., 2017, 19, 26606-26620.

25 S. Grieshammer, Phys. Chem. Chem. Phys., 2018, 20, 19792-19799.

26 C. L. Perkins, M. A. Henderson, C. H. F. Peden and G. S. Herman, J. Vac. Sci. Technol., A, 2001, 19, 1942-1946.

27 D. Arifin, A. Ambrosini, S. A. Wilson, B. Mandal, C. L. Muhich and A. W. Weimer, Int. J. Hydrogen Energy, 2020, 45, 160-174. 